\title{
Nutrition and the circadian system
}

\author{
Gregory D. M. Potter ${ }^{1 *}$, Janet E. Cade ${ }^{2}$, Peter J. Grant ${ }^{3}$ and Laura J. Hardie ${ }^{1}$ \\ ${ }^{1}$ LIGHT Laboratories, Division of Epidemiology and Biostatistics, University of Leeds, Leeds LS2 9JT, UK \\ ${ }^{2}$ Nutritional Epidemiology Group, School of Food Science and Nutrition, University of Leeds, Leeds LS2 9JT, UK \\ ${ }^{3}$ LIGHT Laboratories, Division of Cardiovascular \& Diabetes Research, University of Leeds, Leeds LS2 9JT, UK
}

(Submitted 11 February 2016 - Final revision received 15 March 2016 - Accepted 26 April 2016 - First published online 25 May 2016)

\section{Abstract}

The human circadian system anticipates and adapts to daily environmental changes to optimise behaviour according to time of day and temporally partitions incompatible physiological processes. At the helm of this system is a master clock in the suprachiasmatic nuclei (SCN) of the anterior hypothalamus. The SCN are primarily synchronised to the 24-h day by the light/dark cycle; however, feeding/fasting cycles are the primary time cues for clocks in peripheral tissues. Aligning feeding/fasting cycles with clock-regulated metabolic changes optimises metabolism, and studies of other animals suggest that feeding at inappropriate times disrupts circadian system organisation, and thereby contributes to adverse metabolic consequences and chronic disease development. 'High-fat diets' (HFD) produce particularly deleterious effects on circadian system organisation in rodents by blunting feeding/fasting cycles. Time-of-day-restricted feeding, where food availability is restricted to a period of several hours, offsets many adverse consequences of HFD in these animals; however, further evidence is required to assess whether the same is true in humans. Several nutritional compounds have robust effects on the circadian system. Caffeine, for example, can speed synchronisation to new time zones after jetlag. An appreciation of the circadian system has many implications for nutritional science and may ultimately help reduce the burden of chronic diseases.

Key words: Chrononutrition: Metabolism: Obesity: Time-restricted feeding

Life is exposed to relatively predictable daily changes in the environment, the most conspicuous of which is the daily light/ dark (LD) cycle. Endogenous circadian (approximately 24 h) timing systems have evolved in organisms in response to daily cycles of abiotic (such as temperature cycles) and biotic factors (such as food availability cycles) to generate circadian rhythms in behaviour and physiology to anticipate and adapt to these fluctuations and temporally compartmentalise incompatible biological processes, such as anabolism and catabolism ${ }^{(1)}$. The circadian system therefore primes organisms to feed at specific times, and restricting food access to times at which feeding is typically low in model organisms produces many deleterious health consequences. Fruit flies fed at the 'wrong' time, for example, produce fewer eggs ${ }^{(2)}$, and mice fed during the light period only - the rest period for these nocturnal rodents - are prone to diabetes, the metabolic syndrome, obesity, and even impaired cognitive function ${ }^{(3-6)}$.

The circadian system comprises networks of molecular clocks throughout body tissues. Although circadian rhythms are autonomous, self-sustaining and temperature compensated, the circadian system has remarkable plasticity, and feeding can modify circadian rhythms from the molecular to behavioural level ${ }^{(7,8)}$. Indeed, peripheral tissue clocks such as the liver clock are particularly sensitive to the composition and timing of food consumed. Disorganisation of the circadian system and loss of timing relationships between circadian rhythms in particular are thought to contribute to the development of certain chronic diseases $^{(5)}$. Hence, appropriate nutrition, where energy intake is aligned with energy expenditure and clear feeding/ fasting cycles are synchronised with clock-regulated metabolic changes, helps maintain robust behavioural and physiological circadian rhythms and health ${ }^{(9)}$.

Relatively recent environmental changes have predisposed many individuals to circadian system disruption. The advent of artificial lighting, jetlag induced by high-speed trans-meridian travel, shift work and around-the-clock access to energy-dense food are but a few factors that may conspire to disorganise the circadian system, and thereby adversely affect the health of people in modern societies ${ }^{(7,10,11)}$.

The purposes of this review were therefore to introduce the circadian system, highlight its influences on physiological responses to feeding, show how feeding in turn influences the circadian system and to provide implications for nutritionists and directions for future research.

Abbreviations: CLOCK, circadian locomotor output cycles kaput; FAA, food anticipatory activity; HFD, high-fat diet; SCN, suprachiasmatic nuclei; SIRT, SIRTUIN; TRF, time-of-day-restricted feeding.

* Corresponding author: G. D. M. Potter, email umgdmp@leeds.ac.uk 


\section{The hierarchical circadian system}

\section{Central and peripheral clocks}

The paired suprachiasmatic nuclei (SCN) in the anterior hypothalamus orchestrate circadian rhythms throughout body tissues using autonomic, behavioural and humoral mechanisms ${ }^{(12,13)}$. SCN cells contain cell-autonomous molecular clocks based on negative feedback loops that generate approximately 24-h rhythms in 'clock' gene transcription ${ }^{(14)}$ (Fig. 1). As transcription factors, clock genes temporally segregate incompatible cellular processes by regulating the transcription of myriad clock-controlled genes, many of which are enriched for metabolic functions, and the same molecular clocks present in the SCN regulate rhythmic cellular processes in tissues throughout the body ${ }^{(15)}$. That over half of protein-coding genes in mice have been shown to exhibit circadian transcription in certain conditions ${ }^{(16)}$, and large proportions of proteins and metabolites follow suit ${ }^{(17,18)}$, exemplifies the importance of clock control in metabolism. Post-transcriptional clock protein regulation confers another level of tissuespecific metabolic control ${ }^{(19-22)}$. Recently discovered nontranscriptional rhythms in peroxiredoxins, redox-sensitive proteins, are ubiquitous among organisms of all kingdoms, but how these are integrated with clock gene feedback loops is little understood ${ }^{(23)}$.

In the absence of time cues, the human circadian system has a period of approximately $24 \cdot 2 \mathrm{~h}^{(24)}$ and must therefore be re-set (entrained) daily to the 24-h day. The SCN are primarily entrained by light via a monosynaptic pathway from intrinsically photosensitive retinal ganglion cells in the inner retinae to the $\mathrm{SCN}^{(25)}$. In turn, a multisynaptic pathway from the SCN to the pineal gland is a major route by which photoperiodic information is disseminated ${ }^{(26)}$. During darkness, the pineal gland synthesises melatonin, a hormone that increases sleep propensity and acts on its widely expressed receptors to provide photoperiodic information, and contributes to synchronisation of circadian rhythms in other tissues $^{(27)}$. Dim light melatonin onset (DLMO) can therefore be used as a proxy for the onset of the biological night in humans, with melatonin offset in the morning corresponding to the start of the biological day.

In addition to melatonin, the SCN help maintain appropriate phase relationships among peripheral clocks by regulation of other humoral factors - for example, the SCN produce their own secretions to support synchronisation of clocks in other tissues $^{(28-30)}$. Further SCN secretions also contribute to the rhythmic release of hormones such as glucocorticoids by other tissues $^{(31)}$, and glucocorticoids are particularly important entraining agents for many peripheral clocks. The demonstration that glucocorticoid receptor activation restores approximately $60 \%$ of rhythmic gene transcripts in the mouse liver exemplifies this ${ }^{(32)}$. Another mechanism by which the SCN synchronise clocks throughout tissues is by regulating the circadian body temperature rhythm, as molecular clocks can be entrained by circadian temperature fluctuations by way of the heat shock pathway ${ }^{(33)}$.

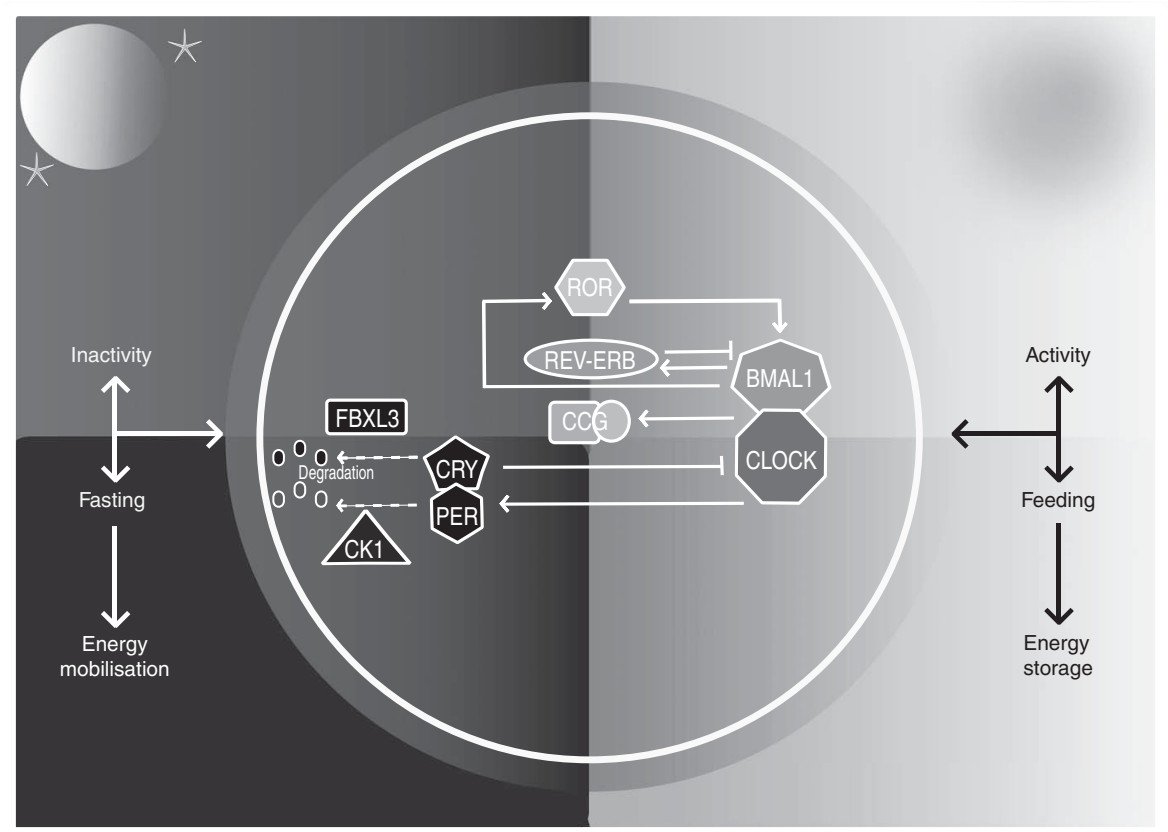

Fig. 1. The mammalian circadian clock. The molecular clock consists of 'clock' genes that form negative-feedback loops. The transcription factors circadian locomotor output cycles kaput (CLOCK) and brain and muscle aryl hydrocarbon receptor nuclear translocator-like 1 (BMAL1) heterodimerise and activate clock-controlled genes (CCG). On activation by CLOCK-BMAL1, cryptochrome (CRY) 1-2 and period (PER) 1-3 proteins accumulate in the cytosol, multimerise and translocate into the nucleus and form inhibitory complexes, repressing CLOCK-BMAL1 and terminating CRY1-2 and PER1-3 transcription during the rest phase. As the rest phase progresses, PER-CRY complexes are degraded by F-box/LRR-repeat protein 3 (FBXL3), casein kinase 1 (CK1) $\varepsilon$ and CK1 $\delta$. Inhibition of CLOCK-BMAL1 activity ends, completing the negative feedback loop. Auxiliary feedback loops are antiphasic to the core loop and regulate $B M A L 1$ transcription. The nuclear receptors reverse-erythroblastosis (REV-ERB) $a$ and $\beta$ repress $B M A L 1$ transcription, whereas RAR-related orphan receptor (ROR) $a$ activates $B M A L 1$ transcription. Auxiliary feedback loops add robustness, among other roles. 
The circadian system readies for feeding during the active phase

As it does with physical activity, the circadian system readies the body for daytime feeding. Human gastric emptying and gastrointestinal motility rates peak in the morning ${ }^{(34,35)}$, and studies in rodents have shown that clock regulation of bile acids and nutrient transporters optimises digestion during the active phase $\mathrm{e}^{(36,37)}$. Furthermore, daily rhythms in the gut microbiota of mice and humans fulfil time-of-day-specific functions, enhancing energy metabolism during the active phase and favouring detoxification during the rest phase ${ }^{(38)}$. The microbiota and circadian system have a complex bidirectional relationship, as disruption of the molecular clock disorganises rhythmic changes in the gut microbiota $^{(39)}$, and germ-free mice have altered clock gene expression ${ }^{(40)}$. Related to such changes, there are circadian rhythms in blood concentrations of many nutrients, such as glucose and lipids ${ }^{(41)}$. An important implication of circadian regulation of the gastrointestinal system is the importance of considering timing of nutritional tests, as exemplified by the recent demonstration that food allergy test results are contingent on the time of day ${ }^{(42)}$.

The circadian system promotes energy substrate storage in appropriate tissues during the day. Insulin sensitivity has a bimodal daily peak during the active phase ${ }^{(43)}$, and appetite for most foods is clock-controlled and lowest in the morning, perhaps to allow consolidated sleep despite diminishing energy availability $^{(44)}$. Diet-induced thermogenesis too has a circadian rhythm that peaks in the morning ${ }^{(45)}$. These changes may be of particular relevance to the obesity epidemic, as they suggest that delayed bedtimes increase time for food consumption when appetite is high, and that consuming a higher proportion of dietary energy in the morning might encourage a negative energy balance, the principle determinant of decreasing body mass.

\section{Feeding entrainment of clocks}

Although the SCN clocks are primarily entrained by light, timeof-day-restricted feeding (TRF) studies, where food availability is restricted to a period of several hours, have shown that peripheral clocks are predominantly responsive to feeding. Indeed, rest phase TRF inverts gene expression profiles in many peripheral tissues including the heart, kidney, liver, pancreas, adipose tissue and the gastrointestinal $\operatorname{tract}^{(7,46-48)}$. The time course of this entrainment varies depending on the organs in question, with the liver clock responding to feeding particularly rapidly. As a result, peripheral tissue rhythms can be uncoupled from SCN rhythms ${ }^{(7)}$. Interestingly, feeding shifts the liver clock more rapidly in SCN-lesioned mice, suggesting that the SCN counters internal desynchronisation - the loss of appropriate phase relationships between clocks that is thought to contribute to metabolic aberrations ${ }^{(49)}$. During ad libitum conditions, TRF does not appear to affect the phase of the SCN clock; however, the SCN clock phase may respond somewhat to TRF combined with energy restriction ${ }^{(50)}$. Although few studies on the effects of TRF on the human circadian system have been published, circadian rhythms in core body temperature and heart rate were advanced after $3 \mathrm{~d}$ of morning $v$. evening TRF in healthy young men ${ }^{(51)}$.

\section{Coupling between metabolism and clocks}

Feeding entrainment of tissue clocks is predicated on reciprocal relationships between molecular clocks and metabolic sensors and regulators ${ }^{(52)}$. Feeding/fasting cycles produce changing nutrient availability, and hence periodic phosphorylation of energy sensors such as 5' AMP-activated protein kinase (AMPK), which promotes ATP production during reduced energy availability, and mechanistic target of rapamycin (mTOR), which promotes anabolic processes during increased energy availability. These regulators are coupled to molecular clock components, which in turn influence myriad metabolic processes integral to nutrient homoeostasis. AMPK, for example, phosphorylates and destabilises cryptochrome (CRY) 1 in peripheral cells ${ }^{(53)}$ and interacts with SIRTUIN (SIRT) 1. In turn, SIRT1 modulates transcription factors including period (PER) $2^{(54)}$ as well as the ventromedial hypothalamic clock, a brain region that contributes to regulation of the circadian rhythm in feeding behaviour ${ }^{(55)}$. SIRT1 is one of a family of deacetylase enzymes that have many roles in metabolic regulation, and SIRT1 and SIRT6 appear to be particularly important to temporal partitioning of metabolism by controlling the transcription of distinct sets of genes with circadian expression profiles, with SIRT6 regulating the rhythmic transcription of genes involved in cholesterol and fatty acid (FA) metabolism ${ }^{(56)}$.

Both tissue-specific and whole-body genetic disruption of the molecular clock produce diverse metabolic aberrations ${ }^{(57,58)}$, and the molecular clock partly mediates beneficial effects of some nutritional interventions, such as the longevity-promoting effects of energy restriction ${ }^{(59)}$. These findings support recent observational studies that have associated SNP in clock genes with various facets of metabolic health. Regarding circadian locomotor output cycles kaput (CLOCK), for example, CLOCK SNP have been associated with non-alcoholic steatohepatitis, the metabolic syndrome, small dense LDL levels, obesity and diabetes $^{(60-64)}$. Perhaps the most studied of these associations is that of obesity: to date, eight common $C L O C K$ SNP have been linked to obesity and three have been associated with energy intakes ${ }^{(65)}$. Results of such small, candidate-gene association studies need support from large, unbiased, genome-wide association studies, however.

\section{Food anticipatory activity and food-entrainable oscillators}

Coupling between nutrient availability and the circadian system is also evident at the behavioural level. TRF in animals such as rats produces food anticipatory activity (FAA) - food-seeking behaviour at times during which food procurement is most likely. FAA is goal-directed towards places where food is available, and may thus be an adaptive strategy to enhance foraging success ${ }^{(8)}$. Indeed, FAA is accentuated during energy restriction. As FAA is entrainable and persists 
during several days of food deprivation, FAA appears to be a true circadian rhythm.

Interestingly, FAA persists both following SCN ablation ${ }^{(66)}$ and disruption of the positive and negative arms of the molecular clock $^{(67)}$; therefore, the food-entrainable oscillators thought to underlie FAA must reside elsewhere. Candidate oscillators comprise various brain structures (including the cerebellum, dorsomedial nuclei, and dorsal striatal and mesocorticolimbic circuits $^{(68-70)}$ ), neurochemical pathways (including dopaminergic and melanocortinergic signalling ${ }^{(71,72)}$ ) and hormonal signals (including ghrelin and orexins ${ }^{(73)}$ ).

\section{Eating patterns: feeding/fasting matters}

As metabolic rhythms are intertwined with nutrient availability, clear feeding/fasting cycles consolidate robust metabolic and behavioural rhythms. High-fat diets (HFD) blunt feeding/fasting cycles in mice, increasing the proportion of energy consumed during the rest phase, and hence dampen circadian rhythms in clock genes ${ }^{(74,75)}$. Consistent with this, expression of adipose tissue clock genes such as PER2 is increased following weight loss in humans ${ }^{(76)}$. Ad libitum access to HFD consistently and rapidly produces obesity in many animals, and endocrine rhythms are similarly blunted in obese humans ${ }^{(77)}$. Whether obesity precedes dampened circadian rhythms has been contentious, but recent evidence indicates that HFD induce rapid re-organisation of gene transcription rhythms before overt increases in adiposity in mice ${ }^{(78)}$.

Compared with ad libitum feeding, TRF offsets HFD-induced blunted feeding rhythms in mice, and the result is superior metabolic health, including reduced adiposity, despite similar energy intakes ${ }^{(75)}$. Comprehensive recent experiments have shown that, despite similar energy intakes and locomotor activity, various TRF schedules are beneficial during different nutritional 'challenges', such as HFD and high-fructose diets, and that beneficial metabolic effects of TRF are proportional to fasting duration ${ }^{(9)}$. During HFD feeding, TRF produces nutrient sensor profiles (including AMPK and mTOR) that are more similar to mice fed normal chow ${ }^{(75)}$. Furthermore, TRF counters HFD-induced reductions in cyclical changes in the gut microbiota, and stool metabolite analyses suggest that this effect of TRF contributes to metabolic health benefits of $\mathrm{TRF}^{(79)}$. These studies used male C57/BL6 mice, animals with a particular susceptibility to diet-induced obesity. As such, it may be premature to extrapolate these findings to humans. Nevertheless, recent research found that eight obese adults with habitual eating periods exceeding $14 \mathrm{~h}$ experienced sustained weight loss and improved sleep when consumption of energy-containing foods and drinks was restricted to an 11 -h period each day ${ }^{(80)}$. The latter study was clearly limited by its sample size, however.

In contrast to the beneficial effects of TRF during HFD feeding, TRF may not confer such striking metabolic advantages when mice are fed normal chow ${ }^{(75)}$. The same may be true among lean humans consuming typical diets. Among fifteen healthy young adults, a cross-over trial found that evening TRF increased fasting glycaemia and impaired glucose tolerance $v$. an isoenergetic diet comprising three meals throughout the day ${ }^{(81)}$. Another study of the same design associated TRF with increased hunger, blood pressure and cholesterol ${ }^{(82)}$. However, findings may have been confounded by circadian variations in these parameters, as measures were taken at different times of the day.

Although not described as TRF studies, breakfast skipping is conceptually akin to TRF. In a larger study of overweight and obese adults, breakfast skipping did not influence responses to weight-loss diets ${ }^{(83)}$, and a careful study in lean young adults found that one of the only effects of 6 weeks of breakfast omission was increased afternoon glycaemic variability $^{(84)}$. Subsequent research using the same protocol in obese adults also found few differences between groups, although insulin sensitivity was higher in breakfast eaters ${ }^{(85)}$. It is possible that breakfast omission altered the timing of peak insulin sensitivity, however. Therefore, it appears that TRF may not benefit metabolic health in all contexts. Certainly, further studies with larger sample sizes are needed. Important questions remain unanswered, such as what is the optimal TRF period and meal frequency, and under what circumstances?

\section{Time-of-day-restricted feeding: meal timing matters}

One factor that may be relevant to the efficacy of TRF is meal timing. Mice fed HFD during the rest phase tended to gain more fat mass than mice fed HFD during the active phase ${ }^{(4)}$. Similarly, mice fed normal chow during the rest phase also gained more fat mass than mice fed during the active phase. Rest phase TRF also altered clock and metabolic gene expression profiles in peripheral tissues, blunted corticosterone rhythm amplitudes, reduced energy expenditure despite comparable locomotor activity and reduced lipid oxidation within $9 \mathrm{~d}^{(6)}$. It is possible that deleterious metabolic effects of rest phase TRF are related to misalignment between energy intake and energy expenditure. Clock gene mutations alter circadian rhythm periods in organisms including humans ${ }^{(86)}$, and a transgenic bPER1 mutation in mice increases obesity risk by advancing peak feeding time relative to peak daily energy expenditure. Subsequently using TRF to synchronise feeding with peak energy expenditure mitigates obesity development in these animals ${ }^{(87)}$.

Ramadan confines eating to the rest phase and modifies circadian rhythms in hormone secretion - for example, the timing of the morning rise in cortisol and night-time melatonin peak are both delayed ${ }^{(88)}$. Some results of Ramadan studies appear to contradict rodent TRF study findings, however. Meta-analysis of thirty-five observational studies found a mean reduction in body mass of $1.24 \mathrm{~kg}$ during Ramadan, with differences between ethnicities and greater reductions in men. No effects on dietary macronutrient proportions were observed, and fasting duration was not associated with body mass changes ${ }^{(89)}$. It was not possible to evaluate body composition, however, and carefully controlled human TRF experiments are needed to determine whether large differences in TRF timing produce similarly large metabolic changes to those seen in mice. 


\section{Time-of-day-restricted feeding: nutrient and energy} distribution timing matters

We refer to nutrient intake timing as the timing of ingestion of specific nutrients and the distribution of energy assigned to eating occasions when the timing of eating occasions is otherwise similar. Studies of mice show that high-fat meal consumption at the end of the active phase increases adiposity, insulin, leptin, and triacylglycerolaemia $v$. consumption at the beginning of the active phase ${ }^{(90)}$. Similarly, restricting fructose access to the rest phase increases adiposity and insulin resistance in comparison with restricting access to the active phase $^{(91)}$

In overweight and obese women matched for energy intakes, those who consumed a larger proportion of daily energy early in the day lost more weight than those consuming more later in the day ${ }^{(92)}$, consistent with other findings that earlier lunch consumption is associated with greater weight loss after 20 weeks $^{(93)}$. Similar associations have since been reported in severely obese adults following bariatric surgery ${ }^{(94)}$. As diet-induced thermogenesis peaks in the morning, and breakfast consumption is associated with higher subsequent non-exercise activity thermogenesis, and hence energy expenditure $^{(84)}$, it is plausible that assigning more of daily energy expenditure to earlier meals may encourage a negative energy balance during hypoenergetic diets. Further studies on how meal composition and energy availability affect responses to TRF will be valuable.

\section{Eating patterns: consistency matters}

Finally, eating patterns are very inconsistent in some adults ${ }^{(80)}$, and this may be relevant to metabolic health. In mice, fixing TRF to a 12-h period during twice-weekly 6-h LD cycle advances might be expected to uncouple LD cycle-entrained SCN rhythms from feeding-entrained peripheral clock rhythms and produce corresponding metabolic disorder. In these conditions, however, TRF mitigated the obesogenic effects of LD cycle shifts observed in ad libitum-fed mice, despite similar energy intakes. Hence, meal regularity and not just its timing relative to activity may be important to metabolic benefits of $\mathrm{TRF}^{(95)}$. The mechanisms by which regular feeding schedules offset obesity in this study are unclear, however, and similar studies in humans are necessary to determine whether these findings are applicable to populations such as shift workers. It will also be interesting to clarify whether TRF needs to be implemented daily to be beneficial; some evidence suggests otherwise ${ }^{(96)}$.

Together, it appears that TRF may be a promising way to improve metabolic health in overweight and obese individuals. Consistent meal patterns and consuming meals shortly after physical activity may help optimise metabolic health. Furthermore, allocating a higher proportion of energy intake to earlier meals may promote a lower energy balance when diets are matched for energy intake. Nevertheless, many questions remain. It is important to determine how effective different TRF schedules are compared to one another and what factors determine inter-individual variability in responses.

\section{Nutrient composition modifies clocks}

The compositions of foods have been shown to influence many different circadian rhythms in rodents, from gene expression profiles to behavioural rhythms. HFD have sometimes but not always been found to influence peripheral tissue clock gene expression profiles in mice studies ${ }^{(74,97)}$, and these discrepancies may have resulted from factors including diet composition. In support of this contention, higher-protein, lower-carbohydrate chow advanced expression rhythms of multiple clock genes in the kidneys and livers of mice, and increased mean expressions of brain and muscle aryl hydrocarbon receptor nuclear translocator-like 1 (Bmal1) and Cry1 in comparison with standard chow ${ }^{(98)}$. In humans, switching participants from higher-carbohydrate (55\%) and lower-fat (30\%) diets to isoenergetic lower-carbohydrate (40\%) and higher-fat (45\%) diets delayed and increased the amplitude of cortisol rhythms, changed inflammatory and metabolic gene expression profiles and altered PER gene expression rhythms in monocytes ${ }^{(99)}$.

In addition to the proportions of dietary energy coming from the macronutrients influencing peripheral clocks, individual nutrients may influence the circadian system, even within certain types of nutrients. Using FA to exemplify this, palmitate, the most abundant SFA in animals, and DHA, a PUFA found plentifully in fish, differentially affected Bmal1 expression in a murine hypothalamic cell line ${ }^{(100)}$. Moreover, manipulating dietary DHA and EPA content shifts liver clock gene expression profiles in mice in vivo ${ }^{(101)}$.

There are also several non-essential dietary compounds consistently shown to influence the circadian system. Alcohol is widely consumed in many societies and appears to be particularly disruptive to molecular, endocrine and behavioural circadian rhythms in humans and other animals ${ }^{(102-106)}$. Caffeine, the most-used psychoactive compound worldwide, is present in many foods and beverages and influences the amplitudes and phases of peripheral tissue clock gene expression rhythms in mice ${ }^{(107)}$. Evening caffeine consumption delays the human circadian system in vivo and lengthens clock gene expression periods in vitro ${ }^{(108)}$. Hence, careful use of caffeine can expedite circadian rhythm entrainment following jetlag ${ }^{(109)}$. However, even if subjective sleepiness is unaffected by its ingestion, caffeine impairs sleep following jetlag ${ }^{(110)}$. Caffeine has also been studied for efficacy in entraining individuals with chronic circadian system dysfunction. In a small study of blind individuals with non-24-h sleep/wake rhythm disorder, a disorder where light fails to synchronise the circadian system with the 24-h d, $150 \mathrm{mg}$ of morning caffeine was insufficient to entrain circadian rhythms ${ }^{(111)}$. Dietary polyphenols are another group of compounds consistently shown to influence both molecular and behavioural circadian rhythms in some $\operatorname{animals}^{(112,113)}$, and other novel nutritional supplements such as dietary polyamines ${ }^{(114)}$ phase-shift the circadian system in rodents. Further research is needed to see whether such compounds might be useful in humans, however; if they are, what are the best times to consume them to maximise their impact, and what are the dose-response and phase-response curves of these compounds? 


\section{Conclusions and directions for future research}

Growing interest in nutrition and the circadian system has produced many insights into the reciprocal relationships between the two in recent years. Findings from these studies have many implications. When assessing nutritional status and the efficacy of nutritional interventions, for example, test timing is an important consideration. More specifically, physiological measures should be taken relative to internal time (DLMO, for example) where feasible. Related to this, chronotype classifies individuals into morning or evening types according to their preference for when to be active and when to sleep. Where laboratory measures of internal time are impractical, chronotype can be estimated by simple questionnaires such as the Morningness-Eveningness Questionnaire and the Munich Chronotype Questionnaire Test ${ }^{(115,116)}$. As chronotype influences the times at which various physiological processes are optimised, consideration of chronotype will be important for personalised nutrition recommendations. Recent studies have also begun exploring how clock gene SNP may influence responses to dietary interventions ${ }^{(117)}$, and ultimately knowledge of circadian system gene variants may also help inform personalised nutrition.

Pressing questions remain unanswered, and there is a glaring need for human studies addressing these. Regarding eating patterns, whether TRF can accelerate entrainment in populations experiencing circadian disruption is a question of relevance to many. With respect to specific foods and supplements, are there dietary interventions with consistently beneficial effects on sleep? It is known that the composition of human breast milk varies daily ${ }^{(118)}$, and perhaps infant formulae should reflect this.

Continuing collaboration between chronobiologists and nutritionists will further clarify interactions between nutrition and the circadian system, and ultimately has the potential to reduce the prevalence and burden of chronic diseases.

\section{Acknowledgements}

G. D. M. P. is supported by a Medical Research Council Doctoral Training Grant. P. J. G. is supported by a EuRhythDia grant (number 278397). J. E. C. and L. J. H. are supported by a Medical Research Council grant (number G1100235/1).

G. D. M. P. wrote the manuscript; J. E. C., P. J. G. and L. J. H. provided constructive feedback at all stages of its preparation.

The authors declare that there are no conflicts of interest.

\section{References}

1. Bass J \& Takahashi JS (2010) Circadian integration of metabolism and energetics. Science 330, 1349-1354.

2. Xu K, DiAngelo JR, Hughes ME, et al. (2011) The circadian clock interacts with metabolic physiology to influence reproductive fitness. Cell Metab 13, 639-654.

3. Loh DH, Jami SA, Flores RE, et al. (2015) Misaligned feeding impairs memories. Elife $\mathbf{4}$, e09460.

4. Arble DM, Bass J, Laposky AD, et al. (2009) Circadian timing of food intake contributes to weight gain. Obesity (Silver Spring) 17, 2100-2102.
5. Mukherji A, Kobiita A, Damara M, et al. (2015) Shifting eating to the circadian rest phase misaligns the peripheral clocks with the master SCN clock and leads to a metabolic syndrome. Proc Natl Acad Sci U S A 112, E6691-E6698.

6. Bray MS, Ratcliffe WF, Grenett MH, et al. (2013) Quantitative analysis of light-phase restricted feeding reveals metabolic dyssynchrony in mice. Int J Obes (Lond) 37, 843-852.

7. Damiola F, Le Minh N, Preitner N, et al. (2000) Restricted feeding uncouples circadian oscillators in peripheral tissues from the central pacemaker in the suprachiasmatic nucleus. Genes Dev 14, 2950-2961.

8. Boulos Z, Rosenwasser AM \& Terman M (1980) Feeding schedules and the circadian organization of behavior in the rat. Behav Brain Res 1, 39-65.

9. Chaix A, Zarrinpar A, Miu P, et al. (2014) Time-restricted feeding is a preventative and therapeutic intervention against diverse nutritional challenges. Cell Metab 20, 991-1005.

10. Moreno CR, Vasconcelos S, Marqueze EC, et al. (2015) Sleep patterns in Amazon rubber tappers with and without electric light at home. Sci Rep 5, 14074.

11. Sack RL, Auckley D, Auger RR, et al. (2007) Circadian rhythm sleep disorders: part I, basic principles, shift work and jet lag disorders. An American Academy of Sleep Medicine review. Sleep 30, 1460-1483.

12. Ralph MR, Foster RG, Davis FC, et al. (1990) Transplanted suprachiasmatic nucleus determines circadian period. Science 247, 975-978.

13. Silver R, LeSauter J, Tresco PA, et al. (1996) A diffusible coupling signal from the transplanted suprachiasmatic nucleus controlling circadian locomotor rhythms. Nature 382, 810-813.

14. Welsh DK, Takahashi JS \& Kay SA (2010) Suprachiasmatic nucleus: cell autonomy and network properties. Annu Rev Physiol 72, 551-577.

15. Partch CL, Green CB \& Takahashi JS (2014) Molecular architecture of the mammalian circadian clock. Trends Cell Biol 24, 90-99.

16. Patel VR, Ceglia N, Zeller M, et al. (2015) The pervasiveness and plasticity of circadian oscillations: the coupled circadianoscillators framework. Bioinformatics 31, 3181-3188.

17. Reddy AB, Karp NA, Maywood ES, et al. (2006) Circadian orchestration of the hepatic proteome. Curr Biol 16, 1107-1115.

18. Eckel-Mahan KL, Patel VR, Mohney RP, et al. (2012) Coordination of the transcriptome and metabolome by the circadian clock. Proc Natl Acad Sci U S A 109, 5541-5546.

19. Cardone L, Hirayama J, Giordano F, et al. (2005) Circadian clock control by SUMOylation of BMAL1. Science 309 , 1390-1394

20. Zhang R, Lahens NF, Ballance HI, et al. (2014) A circadian gene expression atlas in mammals: implications for biology and medicine. Proc Natl Acad Sci U S A 111, 16219-16224.

21. Asher G, Reinke H, Altmeyer M, et al. (2010) Poly(ADPribose) polymerase 1 participates in the phase entrainment of circadian clocks to feeding. Cell 142, 943-953.

22. Hirayama J, Sahar S, Grimaldi B, et al. (2007) CLOCKmediated acetylation of BMAL1 controls circadian function. Nature 450, 1086-1090.

23. O'Neill JS, van Ooijen G, Dixon LE, et al. (2011) Circadian rhythms persist without transcription in a eukaryote. Nature 469, 554-558.

24. Czeisler CA, Duffy JF, Shanahan TL, et al. (1999) Stability, precision, and near-24-hour period of the human circadian pacemaker. Science 284, 2177-2181. 
25. Berson DM, Dunn FA \& Takao M (2002) Phototransduction by retinal ganglion cells that set the circadian clock. Science 295, 1070-1073.

26. Moore RY (1996) Neural control of the pineal gland. Behav Brain Res 73, 125-130.

27. Zawilska JB, Skene DJ \& Arendt J (2009) Physiology and pharmacology of melatonin in relation to biological rhythms. Pharmacol Rep 61, 383-410.

28. Kramer A, Yang FC, Snodgrass P, et al. (2003) Regulation of daily locomotor activity and sleep by hypothalamic EGF receptor signalling. Science 294, 2511-2515.

29. Cheng MY, Bullock CM, Li C, et al. (2002) Prokineticin 2 transmits the behavioural circadian rhythm of the suprachiasmatic nucleus. Nature 417, 405-410.

30. Kraves S \& Weitz CJ (2006) A role for cardiotrophin-like cytokine in the circadian control of mammalian locomotor activity. Nat Neurosci 9, 212-219.

31. Kalsbeek A, van Heerikhuize JJ, Wortel J, et al. (1996) A diurnal rhythm of stimulatory input to the hypothalamopituitary-adrenal system as revealed by timed intrahypothalamic administration of the vasopressin V1 antagonist. J Neurosci 16, 5555-5565.

32. Reddy AB, Maywood ES, Karp NA, et al. (2007) Glucocorticoid signaling synchronizes the liver circadian transcriptome. Hepatology 45, 1478-1488.

33. Buhr ED, Yoo SH \& Takahashi JS (2010) Temperature as a universal resetting cue for mammalian circadian oscillators. Science 330, 379-385.

34. Goo RH, Moore JG, Greenberg E, et al. (1987) Circadian variation in gastric emptying of meals in humans. Gastroenterology 93, 515-518.

35. Rao SS, Sadeghi P, Beaty J, et al. (2001) Ambulatory 24-h colonic manometry in healthy humans. Am J Physiol Gastrointest Liver Physiol 280, G629-G639.

36. Han SS, Zhang R, Jain R, et al. (2015) Circadian control of bile acid synthesis by a KLF15-Fgf15 axis. Nat Commun 6, 7231.

37. Hussain MM \& Pan X (2015) Circadian regulation of macronutrient absorption. J Biol Rhythms 30, 459-469.

38. Thaiss CA, Zeevi D, Levy M, et al. (2014) Transkingdom control of microbiota diurnal oscillations promotes metabolic homeostasis. Cell 159, 514-529.

39. Liang X, Bushman FD \& FitzGerald GA (2015) Rhythmicity of the intestinal microbiota is regulated by gender and the host circadian clock. Proc Natl Acad Sci U S A 112, 10479-10484.

40. Leone V, Gibbons SM, Martinez K, et al. (2015) Effects of diurnal variation of gut microbes and high-fat feeding on host circadian clock function and metabolism. Cell Host Microbe 17, 681-689.

41. Morgan L, Arendt J, Owens D, et al. (1998) Effects of the endogenous clock and sleep time on melatonin, insulin, glucose and lipid metabolism. J Endocrinol 157, 443-451.

42. Tanabe K, Kitagawa E, Wada M, et al. (2015) Antigen exposure in the late light period induces severe symptoms of food allergy in an OVA-allergic mouse model. Sci Rep $\mathbf{5}$, 14424.

43. Scheer FA, Hilton MF, Mantzoros CS, et al. (2009) Adverse metabolic and cardiovascular consequences of circadian misalignment. Proc Natl Acad Sci U S A 106, 4453-4458.

44. Scheer FA, Morris CJ \& Shea SA (2013) The internal circadian clock increases hunger and appetite in the evening independent of food intake and other behaviors. Obesity (Silver Spring) 21, 421-423.
45. Morris CJ, Garcia JI, Myers S, et al. (2015) The human circadian system has a dominating role in causing the morning/evening difference in diet-induced thermogenesis. Obesity (Silver Spring) 23, 2053-2058.

46. Stokkan KA, Yamazaki S, Tei H, et al. (2001) Entrainment of the circadian clock in the liver by feeding. Science 291, 490-493.

47. Zvonic S, Ptitsyn AA, Conrad SA, et al. (2006) Characterization of peripheral circadian clocks in adipose tissues. Diabetes 55, 962-970.

48. Hoogerwerf WA, Hellmich HL, Cornelissen G, et al. (2007) Clock gene expression in the murine gastrointestinal tract: endogenous rhythmicity and effects of a feeding regimen. Gastroenterology 133, 1250-1260.

49. Saini C, Liani A, Curie T, et al. (2013) Real-time recording of circadian liver gene expression in freely moving mice reveals the phase-setting behavior of hepatocyte clocks. Genes Dev 27, 1526-1536.

50. Mendoza J, Graff C, Dardente H, et al. (2005) Feeding cues alter clock gene oscillations and photic responses in the suprachiasmatic nuclei of mice exposed to a light/ dark cycle. J Neurosci 25, 1514-1522.

51. Krauchi K, Cajochen C, Werth E, et al. (2002) Alteration of internal circadian phase relationships after morning versus evening carbohydrate-rich meals in humans. J Biol Rhythms 17, 364-376.

52. Eckel-Mahan K \& Sassone-Corsi P. (2013) Metabolism and the circadian clock converge. Physiol Rev 93, 107-135.

53. Lamia KA, Sachdeva UM, DiTacchio L, et al. (2009) AMPK regulates the circadian clock by cryptochrome phosphorylation and degradation. Science 326, 437-440.

54. Asher G, Gatfield D, Stratmann M, et al. (2008) SIRT1 regulates circadian clock gene expression through PER2 deacetylation. Cell 134, 317-328.

55. Orozco-Solis R, Ramadori G, Coppari R, et al. (2015) SIRT1 relays nutritional inputs to the circadian clock through the sf1 neurons of the ventromedial hypothalamus. Endocrinology 156, 2174-2184.

56. Masri S, Rigor P, Cervantes M, et al. (2014) Partitioning circadian transcription by SIRT6 leads to segregated control of cellular metabolism. Cell 158, 659-672.

57. Paschos GK, Ibrahim S, Song WL, et al. (2012) Obesity in mice with adipocyte-specific deletion of clock component Arntl. Nat Med 18, 1768-1777.

58. Turek FW, Joshu C, Kohsaka A, et al. (2005) Obesity and metabolic syndrome in circadian clock mutant mice. Science 308, 1043-1045.

59. Patel SA, Chaudhari A, Gupta R, et al. (2015) Circadian clocks govern calorie restriction-mediated life span extension through BMAL1- and IGF-1-dependent mechanisms. FASEB J 30, 1634-1642.

60. Sookoian S, Castano G, Gemma C, et al. (2007) Common genetic variations in clock transcription factor are associated with nonalcoholic fatty liver disease. World J Gastroenterol 13, 4242-4248.

61. Scott EM, Carter AM \& Grant PJ (2008) Association between polymorphisms in the clock gene, obesity and the metabolic syndrome in man. Int J Obes (Lond) 32, 658-662.

62. Tsuzaki K, Kotani K, Sano Y, et al. (2010) The association of the Clock 3111 T/C SNP with lipids and lipoproteins including small dense low-density lipoprotein: results from the Mima study. BMC Med Genet 11, 150.

63. Sookoian S, Gemma C, Gianotti TF, et al. (2008) Genetic variants of clock transcription factor are associated with individual susceptibility to obesity. Am J Clin Nutr 87, $1606-1615$ 
64. Uemura H, Katsuura-Kamano S, Yamaguchi M, et al. (2015) A variant of the CLOCK gene and related haplotypes are associated with the prevalence of type 2 diabetes in the Japanese population. J Diabetes (epublication ahead of print version 16 September 2015).

65. Valladares M, Obregon AM \& Chaput JP (2015) Association between genetic variants of the clock gene and obesity and sleep duration. J Physiol Biochem 71, 855-860.

66. Stephan FK, Swann JM \& Sisk CL (1979) Anticipation of 24-hr feeding schedules in rats with lesions of the suprachiasmatic nucleus. Behav Neural Biol 25, 346-363.

67. Storch KF \& Weitz CJ (2009) Daily rhythms of foodanticipatory behavioral activity do not require the known circadian clock. Proc Natl Acad Sci U S A 106, 6808-6813.

68. Mendoza J, Pevet P, Felder-Schmittbuhl MP, et al. (2010) The cerebellum harbors a circadian oscillator involved in food anticipation. J Neurosci 30, 1894-1904.

69. Landry GJ, Kent BA, Patton DF, et al. (2011) Evidence for time-of-day dependent effect of neurotoxic dorsomedial hypothalamic lesions on food anticipatory circadian rhythms in rats. PLOS ONE 6, e24187.

70. Verwey M \& Amir S (2009) Food-entrainable circadian oscillators in the brain. Eur J Neurosci 30, 1650-1657.

71. Gallardo CM, Darvas M, Oviatt M, et al. (2014) Dopamine receptor 1 neurons in the dorsal striatum regulate food anticipatory circadian activity rhythms in mice. Elife 3, $\mathrm{e} 03781$.

72. Sutton GM, Perez-Tilve D, Nogueiras R, et al. (2008) The melanocortin-3 receptor is required for entrainment to meal intake. J Neurosci 28, 12946-12955.

73. LeSauter J, Hoque N, Weintraub M, et al. (2009) Stomach ghrelin-secreting cells as food-entrainable circadian clocks. Proc Natl Acad Sci U S A 106, 13582-13587.

74. Kohsaka A, Laposky AD, Ramsey KM, et al. (2007) High-fat diet disrupts behavioral and molecular circadian rhythms in mice. Cell Metab 6, 414-421.

75. Hatori M, Vollmers C, Zarrinpar A, et al. (2012) Timerestricted feeding without reducing caloric intake prevents metabolic diseases in mice fed a high-fat diet. Cell Metab 15 848-860.

76. Pivovarova O, Gogebakan O, Sucher S, et al. (2016) Regulation of the clock genes expression in human adipose tissue by the weight loss. Int J Obes (Lond) (epublication ahead of print version 23 February 2016).

77. Matkovic V, Ilich JZ, Badenhop NE, et al. (1997) Gain in body fat is inversely related to the nocturnal rise in serum leptin level in young females. J Clin Endocrinol Metab 82, 1368-1372.

78. Eckel-Mahan KL, Patel VR, de Mateo S, et al. (2013) Reprogramming of the circadian clock by nutritional challenge. Cell 155, 1464-1478.

79. Zarrinpar A, Chaix A, Yooseph S, et al. (2014) Diet and feeding pattern affect the diurnal dynamics of the gut microbiome. Cell Metab 20, 1006-1017.

80. Gill S \& Panda S. (2015) A smartphone app reveals erratic diurnal eating patterns in humans that can be modulated for health benefits. Cell Metab 22, 789-798.

81. Carlson O, Martin B, Stote KS, et al. (2007) Impact of reduced meal frequency without caloric restriction on glucose regulation in healthy, normal-weight middle-aged men and women. Metabolism 56, 1729-1734.

82. Stote KS, Baer DJ, Spears K, et al. (2007) A controlled trial of reduced meal frequency without caloric restriction in healthy, normal-weight, middle-aged adults. Am J Clin Nutr 85, 981-988.
83. Dhurandhar EJ, Dawson J, Alcorn A, et al. (2014) The effectiveness of breakfast recommendations on weight loss: a randomized controlled trial. Am J Clin Nutr 100, 507-513.

84. Betts JA, Richardson JD, Chowdhury EA, et al. (2014) The causal role of breakfast in energy balance and health: a randomized controlled trial in lean adults. Am J Clin Nutr 100, 539-547.

85. Chowdhury EA, Richardson JD, Holman GD, et al. (2016) The causal role of breakfast in energy balance and health: a randomized controlled trial in obese adults. Am J Clin Nutr 103, 747-756.

86. Toh KL, Jones CR, He Y, et al. (2001) An hPer2 phosphorylation site mutation in familial advanced sleep phase syndrome. Science 291, 1040-1043.

87. Liu Z, Huang M, Wu X, et al. (2014) PER1 phosphorylation specifies feeding rhythm in mice. Cell Rep 7, 1509-1520.

88. Bogdan A, Bouchareb B \& Touitou Y (2001) Ramadan fasting alters endocrine and neuroendocrine circadian patterns. Meal-time as a synchronizer in humans? Life Sci $\mathbf{6 8}$, $1607-1615$.

89. Sadeghirad B, Motaghipisheh S, Kolahdooz F, et al. (2014) Islamic fasting and weight loss: a systematic review and meta-analysis. Public Health Nutr 17, 396-406.

90. Bray MS, Tsai JY, Villegas-Montoya C, et al. (2010) Time-of-day-dependent dietary fat consumption influences multiple cardiometabolic syndrome parameters in mice. Int J Obes (Lond) 34, 1589-1598.

91. Morris M, Araujo IC, Pohlman RL, et al. (2012) Timing of fructose intake: an important regulator of adiposity. Clin Exp Pharmacol Physiol 39, 57-62.

92. Jakubowicz D, Barnea M, Wainstein J, et al. (2013) High caloric intake at breakfast vs. dinner differentially influences weight loss of overweight and obese women. Obesity (Silver Spring) 21, 2504-2512.

93. Garaulet $\mathrm{M}$, Gomez-Abellan $\mathrm{P}$, Alburquerque-Bejar JJ, et al. (2013) Timing of food intake predicts weight loss effectiveness. Int J Obes (Lond) 37, 604-611.

94. Ruiz-Lozano T, Vidal J, de Hollanda A, et al. (2016) Timing of food intake is associated with weight loss evolution in severe obese patients after bariatric surgery. Clin Nutr (epublication ahead of print version 16 February 2016)

95. Oike H, Sakurai M, Ippoushi K, et al. (2015) Time-fixed feeding prevents obesity induced by chronic advances of light/dark cycles in mouse models of jet-lag/shift work. Biochem Biophys Res Commun 465, 556-561.

96. Halberg N, Henriksen M, Soderhamn N, et al. (2005) Effect of intermittent fasting and refeeding on insulin action in healthy men. J Appl Physiol (1985) 99, 2128-2136.

97. Yanagihara H, Ando H, Hayashi $\mathrm{Y}$, et al. (2006) High-fat feeding exerts minimal effects on rhythmic mRNA expression of clock genes in mouse peripheral tissues. Chronobiol Int 23, 905-914.

98. Oishi K, Uchida D \& Itoh N (2012) Low-carbohydrate, high-protein diet affects rhythmic expression of gluconeogenic regulatory and circadian clock genes in mouse peripheral tissues. Chronobiol Int 29, 799-809.

99. Pivovarova O, Jurchott K, Rudovich N, et al. (2015) Changes of dietary fat and carbohydrate content alter central and peripheral clock in humans. J Clin Endocrinol Metab 100, 2291-2302.

100. Greco JA, Oosterman JE \& Belsham DD. (2014) Differential effects of omega-3 fatty acid docosahexaenoic acid and palmitate on the circadian transcriptional profile of clock 
genes in immortalized hypothalamic neurons. Am J Physiol Regul Integr Comp Physiol 307, R1049-R1060.

101. Furutani A, Ikeda Y, Itokawa M, et al. (2015) Fish oil accelerates diet-induced entrainment of the mouse peripheral clock via GPR120. PLOS ONE 10, e0132472.

102. Huang MC, Ho CW, Chen CH, et al. (2010) Reduced expression of circadian clock genes in male alcoholic patients. Alcohol Clin Exp Res 34, 1899-1904.

103. Ando H, Ushijima K, Kumazaki M, et al. (2010) Associations of metabolic parameters and ethanol consumption with messenger RNA expression of clock genes in healthy men. Chronobiol Int 27, 194-203.

104. Filiano AN, Millender-Swain T, Johnson R Jr., et al. (2013) Chronic ethanol consumption disrupts the core molecular clock and diurnal rhythms of metabolic genes in the liver without affecting the suprachiasmatic nucleus. PLOS ONE 8, e71684.

105. Conroy DA, Hairston IS, Arnedt JT, et al. (2012) Dim light melatonin onset in alcohol-dependent men and women compared with healthy controls. Chronobiol Int 29, 35-42.

106. Brager AJ, Ruby CL, Prosser RA, et al. (2010) Chronic ethanol disrupts circadian photic entrainment and daily locomotor activity in the mouse. Alcohol Clin Exp Res 34, 1266-1273.

107. Sherman H, Gutman R, Chapnik N, et al. (2011) Caffeine alters circadian rhythms and expression of disease and metabolic markers. Int J Biochem Cell Biol 43, 829-838.

108. Burke TM, Markwald RR, McHill AW, et al. (2015) Effects of caffeine on the human circadian clock in vivo and in vitro. Sci Transl Med 7, 305ra146.

109. Pierard C, Beaumont M, Enslen M, et al. (2001) Resynchronization of hormonal rhythms after an eastbound flight in humans: effects of slow-release caffeine and melatonin. EurJ Appl Physiol 85, 144-150.
110. Beaumont M, Batejat D, Pierard C, et al. (2004) Caffeine or melatonin effects on sleep and sleepiness after rapid eastward transmeridian travel. J Appl Physiol (1985) 96, 50-58.

111. St Hilaire MA \& Lockley SW (2015) Caffeine does not entrain the circadian clock but improves daytime alertness in blind patients with non-24-hour rhythms. Sleep Med 16, 800-804

112. Ribas-Latre A, Baselga-Escudero L, Casanova E, et al. (2015) Dietary proanthocyanidins modulate BMAL1 acetylation, Nampt expression and NAD levels in rat liver. Sci Rep 5, 10954.

113. Pifferi F, Dal-Pan A, Menaker M, et al. (2011) Resveratrol dietary supplementation shortens the free-running circadian period and decreases body temperature in a prosimian primate. J Biol Rhythms 26, 271-275.

114. Zwighaft Z, Aviram R, Shalev M, et al. (2015) Circadian clock control by polyamine levels through a mechanism that declines with age. Cell Metab 22, 874-885.

115. Horne JA \& Ostberg O (1976) A self-assessment questionnaire to determine morningness-eveningness in human circadian rhythms. Int J Chronobiol 4, 97-110.

116. Roenneberg T, Wirz-Justice A \& Merrow M (2003) Life between clocks: daily temporal patterns of human chronotypes. J Biol Rhythms 18, 80-90.

117. Garcia-Rios A, Gomez-Delgado FJ, Garaulet M, et al. (2014) Beneficial effect of CLOCK gene polymorphism rs1801260 in combination with low-fat diet on insulin metabolism in the patients with metabolic syndrome. Chronobiol Int 31, 401-408.

118. Illnerova H, Buresova M \& Presl J (1993) Melatonin rhythm in human milk. J Clin Endocrinol Metab 77, 838-841. 